La comunicación para el cambio social y el proceso de empoderamiento en una comunidad virtual: el caso del grupo de Facebook Ni Una Menos: Movilización Nacional Ya

Communication for Social Change and the Process of Empowerment in a Virtual Community: The Case of the Facebook Group Ni Una Menos: Movilización Nacional Ya

Es licenciada en Comunicación para el Desarrollo por la Pontificia Universidad Católica del Perú (PUCP) con estudios en la Universidad de Mannheim en Alemania y la Pontificia Universidad Javeriana de Colombia. Fue ganadora del Programa de Apoyo al Desarrollo de Tesis de Licenciatura (PADET) de la PUCP en 2019. Actualmente, trabaja para Inclusiva Consulting, consultora experta en el segmento de la base de la pirámide y los negocios inclusivos. 



\section{La comunicación para el cambio social y el proceso de empoderamiento en una comunidad virtual: el caso del grupo de Facebook Ni Una Menos: Movilización Nacional Ya Communication for Social Change and the Process of Empowerment in a Virtual Community: The Case of the Facebook Group Ni Una Menos: Movilización Nacional Ya}

\section{Valeria Soto Canales}

Facultad de Ciencias y Artes de la Comunicación, Pontificia Universidad Católica del Perú valeria.soto@pucp.pe (https://orcid.org/oooo-0oo2-3911-4270)

Recibido: 31-05-2020 / Aceptado: 10-10-2020

https://doi.org/10.1880o/conexion.202002.007

PALABRAS CLAVE / KEYWORDS

Comunicación para el cambio social, comunidades virtuales, activismo, revelación sensible, empoderamiento, feminismo / communication for social change, virtual communities, activism, sensitive disclosure, empowerment, feminism

\section{RESUMEN}

El presente artículo tiene como objetivo analizar el poder de la interacción comunicativa en comunidades virtuales -específicamente en el caso de la comunidad virtual de Facebook Ni Una Menos: Movilización Nacional Ya- desde el paradigma de la comunicación para el cambio social. Para conseguir dicho objetivo, se aplicó una metodología cualitativa que consistió en un trabajo de campo compuesto por entrevistas a profundidad a 15 integrantes del grupo de Facebook, así como el análisis de contenidos virtuales en medios so- ciales. Producto de este proceso, se pudo observar cómo las características del grupo, creado para el activismo, propiciaron la revelación sensible de testimonios en el portal web, en torno a los cuales se articuló una comunidad virtual de soporte. Como conclusión, este análisis evidenció el poder de la interacción comunicativa virtual para el proceso de empoderamiento individual y colectivo de comunidades vulnerables, como es el caso de las mujeres víctimas de violencia.

\section{ABSTRACT}

The purpose of this article is to analyze the power of communicative interaction in virtual communities, in the case of the virtual community of Facebook Ni Una Menos: Movilización Nacional Ya, from the paradigm of communication for social change. In order to achieve this objective, a qualitative methodology was applied, which consisted of field work composed of in-depth interviews with 15 members of 
the Facebook group, as well as the analysis of virtual contents. As a result of the analysis, it was possible to observe how the characteristics of the group, created for activism, led to the sensitive disclosure of testimonies on the web portal, around which a virtual community of support was articulated. In conclusion, this analysis evidenced the power of virtual communicative interaction for the process of individual and collective empowerment of vulnerable communities, such as women victims of violence.

\section{La comunicación para el cambio social y el proceso de empoderamiento en una comunidad virtual: el caso del grupo de Facebook Ni Una Menos: Movilización Nacional Ya ${ }^{1}$}

\section{Introducción: de un grupo para la movilización social a una comunidad virtual de soporte}

Según la Encuesta Nacional de Relaciones Sociales (ENARES), en el año 2015, el índice de tolerancia social del maltrato a las mujeres en el Perú era del 54.8 \% (Instituto Nacional de Estadística e Informática, 2016, p. 28); esto evidenciaba que la violencia contra la mujer era aún mayoritariamente aceptada. Así, muchas de las mujeres que habían pasado por una experiencia de violencia se sentían solas y únicas víctimas. Su sufrimiento era individual y aislado al no haber -o no poder acceder a- espacios de reconocimiento, información, alerta y apoyo.

En este contexto, en julio de 2015, Arlette Contreras fue agredida en un hotel en Ayacucho por Adriano Pozo. A pesar de que el incidente había sido registrado en cámaras de video y de que existían pruebas contundentes del brutal ataque, la condena en el año 2016 fue de un año de prisión suspendida por «lesiones leves» (América Noticias, 2016, párr. 1). Ello despertó la indignación de la población, pues, una vez más, la justicia le daba la espalda a una víctima de violencia. Este fue el punto de inflexión para que un grupo de mujeres en Lima comenzara a organizarse en internet para responder de forma colectiva frente a la impunidad (Caballero Rojas, 2018) y, tomando como referencia el movimiento del mismo nombre en Argentina, Chile y México, creara el grupo cerrado de Facebook Ni Una Menos: Movilización Nacional Ya, con el fin de planificar una gran movilización que se llevaría a cabo el 13 de agosto de 2016.

Fue así como, en pocas horas, el grupo ya contaba con miles de miembros que, en su mayoría, eran mujeres organizándose rápidamente para la manifestación. De

\footnotetext{
${ }^{1}$ Este artículo es producto de la investigación realizada por la autora -en calidad de estudiante de la Facultad de Ciencias y Artes de la Comunicación de la Pontificia Universidad Católica del Perú- en la tesis de licenciatura El poder de la interacción comunicativa en comunidades virtuales: el caso del grupo de Facebook «Ni una menos, movilización nacional ya» (Soto Canales, 2019).
} 
pronto, una mujer señaló que era necesario «salir del clóset» ${ }^{2}$ de la violencia y compartió su testimonio de violación. De inmediato, recibió una ola de comentarios de apoyo, soporte y admiración; y, a continuación, miles de mujeres replicaron su acción y compartieron también sus experiencias. Así, el grupo se tornó en una comunidad de soporte, a partir del fenómeno de revelaciones sensibles ${ }^{3}$ (Andalibi y Forte, 2018) de testimonios en dicha plataforma online.

En este artículo, se ahondará en el proceso comunicacional que se gestó dentro de esta comunidad y que da luces acerca de componentes del enfoque de la comunicación para el cambio social, como la participación, la apropiación, la concientización y el involucramiento ciudadano, que, al día de hoy, están siendo rearticulados dentro del nuevo escenario tecnológico (Tufte, 2015). Asimismo, se aplicará el enfoque desde el empoderamiento para, a partir de la síntesis de experiencias que validen su potencial como instrumento de cambio desde la ciudadanía, contribuir a la exploración empírica del concepto.

En esta línea, se analizará la interacción comunicativa en este grupo cerrado de Facebook, cuyo sentido fue reconfigurado: no solo es un caso de ciberactivismo, sino de generación de comunidad, en la que se fomenta el empoderamiento individual y colectivo.

\section{Marco teórico: comunidades virtuales para el cambio social}

En los últimos años, se ha generado una profunda transformación tecnológica que está basada en redes horizontales de comunicación interactiva y multidireccional en internet, y que hace posible lo que Castells (2012) denomina la sociedad red, que se establece como la nueva estructura social.

Dentro de ella, una de las principales revoluciones ha sido el surgimiento de los sitios de red social ${ }^{4}$, canales que permiten a los usuarios generar su propio contenido e interactuar con diversos públicos (Carr y Hayes, 2015). Así, se va gestando una infraestructura de la comunicación orientada a los individuos, en la que el principal actor es el «prosumidor», que no solo consume, sino que también produce contenidos, y que pasa de ser un objeto pasivo a ser un sujeto activo en el desarrollo de sus intereses.

Dentro de los sitios de red social, surgen asociaciones de discusión en línea o comunidades virtuales, que son grupos de personas con intereses compartidos que

\footnotetext{
${ }^{2}$ Del inglés coming out, es el proceso por el cual una persona expresa a otros -amigos, familia, etcétera- su orientación sexual o identidad de género (National LGBT Health Education Center, 2018, p. 5).

${ }^{3}$ Traducción propia de sensitive disclosures.

${ }^{4}$ Del inglés social network sites (SNS).
} 
se comunican a través de internet (Butler et al., 2014). Las articula un sentimiento de pertenencia entre los miembros, que se puede manifestar a través de la identificación con las publicaciones, la producción de confianza e, incluso, el soporte emocional (Blanchard y Lynne, 2004; Hsiu-Fen, 2007).

Cabe mencionar que, en países como el Perú, la interacción en línea está limitada por una marcada brecha digital y de uso, que genera que la conformación de estos grupos también se vea afectada por los límites de nivel socioeconómico, origen, nivel educativo, etcétera (Yates y Lockley, 2018). Como se evidencia en datos del Instituto Nacional de Estadística e Informática (2020, pp. 9-10), la población con nivel educativo superior accede más a internet: el $94.7 \%$ de este grupo accede al servicio, mientras que solo el $25 \%$ de la población con educación primaria lo consigue. Considerando estas restricciones y la brecha de acceso digital, en el primer trimestre del año la población usuaria de internet significó el $78.5 \%$ en Lima Metropolitana frente al $23.8 \%$ en el área rural.

A pesar de esta limitación, los medios sociales son una importante herramienta que facilita el desarrollo de los componentes básicos de la teoría de la comunicación para el cambio social: el involucramiento de los agentes y su participación. Así pues, la interacción puede permitir la coordinación entre los propios sujetos para organizarse en función de objetivos, necesidades o intereses.
Asimismo, observamos cómo se manifiesta también el concepto de apropiación, que se refiere a la acción de controlar la creación y el proceso comunicacional que involucra la participación comunitaria (Gumucio-Dagron, 2001/2002). De esta forma, la colectividad misma busca amplificar sus voces, fomentando el debate y el diálogo con algún objetivo y prescindiendo de la comunicación de arriba abajo.

Producto de ello surgen, por ejemplo, las movilizaciones sociales, las cuales refieren a grupos de personas que emprenden manifestaciones colectivas y que, hoy en día, se organizan usualmente desde los nuevos medios de comunicación y la sociedad red. Estas movilizaciones son desencadenadas por una chispa de indignación relacionada con algún acontecimiento concreto. En consecuencia, a través de la condensación emocional, las experiencias individuales de frustración se transforman en una pasión colectiva (Castells, 2012; Gerbaudo, 2012). Así, buscan ejercer presión a través de la ocupación de un espacio urbano y otras acciones articuladas en red, y generan esperanza de cambios.

A través de dinámicas como la mencionada, se evidencia el último proceso del paradigma de la comunicación para el cambio social: la concientización. Esta alude a la necesidad de que determinada comunidad tome conciencia de sí misma y de sus necesidades, y descubra escenarios alternativos que se convierten en 
estrategias para el cambio (Cadiz, 2005; Mendivil Calderón et al., 2015).

Otra de las iniciativas que evidencian la apropiación de los medios comunicacionales es la consolidación de los espacios de soporte en línea, lo que sucedió en el grupo de Facebook objeto de este estudio y Gil Piedra (2017) denomina la consolidación de una «comunidad afectiva e imaginada», en la que se generan dinámicas de solidaridad y empatía a través de la articulación de una gama de biografías individuales que, en su conjunto, afectan al cambio social.

Particularmente, en el caso de las mujeres, el proceso de revelación sensible o la exposición de testimonios cobra especial relevancia, ya que muchas de estas vivencias están normalizadas; gracias a su exposición, se evidencia que sus experiencias no son casos aislados, sino que se articulan de forma colectiva (Garzón Martínez, 2015; Troncoso Pérez y Piper Shafir, 2015).

Además, este proceso genera beneficios en las participantes, como la validación, la identificación con otras, la reducción del aislamiento, el aumento del sentido de pertenencia y el refuerzo de la autoestima (Bazarova et al., 2015). Asimismo, la dinámica propicia una especie de contagio emocional que genera empatía y, por tanto, el reconocimiento de una problemática.

De lo previamente señalado, se evidencia la importancia de la participación para el empoderamiento. El empoderamiento es definido como el proceso por el cual las personas, organizaciones y comunidades ganan control sobre sus vidas con un entendimiento crítico de su entorno (Cano Isaza y Arroyave Álvarez, 2014), que, además, les permite promover cambios en sus contextos comunes.

En el caso del empoderamiento de la mujer, el primer paso es el reconocimiento de la problemática de la dominación masculina, ya que, a través de un proceso reflexivo conjunto, las mujeres pueden cuestionar las prácticas que menoscaban su valor, tomando conciencia y acción respecto a su autoimagen, sus capacidades y sus derechos (Ochman Ikanowicz, 2016).

En el caso de la comunidad de Facebook, ello se evidencia desde el proceso de interacción, tanto online como offline, pues, a través del intercambio de experiencias, apoyo e información, se genera sentido de pertenencia y organización comunitaria (Amichai-Hamburger et al., 2008; Petrič y Petrovčič, 2014), lo que, además, repercute en el plano individual de las personas.

\section{Metodología: adentrándonos en la comunidad virtual}

El trabajo de investigación fue de carácter exploratorio, con un enfoque cualitativo, ya que buscó comprender y profundizar en la perspectiva de las participantes, ahondando en sus experiencias, opiniones y significados (Hernández Sampieri 
et al., 1991/2010). Esta metodología permitió realizar una aproximación empírica del enfoque de la comunicación para el cambio social, cuyos postulados son, en su mayoría, teóricos.

Esta investigación tiene como objetivo analizar la interacción comunicativa generada en la comunidad virtual de Facebook Ni Una Menos: Movilización Nacional Ya para explorar el impacto en las participantes y en su contexto social. Como objetivos secundarios, se encuentran el evaluar las características de la comunidad de Facebook, identificar las dinámicas de interacción comunicativa en el proceso de revelación y determinar el efecto en las participantes.

Acorde con ello, se fue construyendo una metodología de análisis y reflexión flexible para acercarse a las dinámicas comunicativas de cada participante. De este modo, se utilizaron dos herramientas de recojo de información: las entrevistas a profundidad semiestructuradas, de modo que se pudo indagar en las motivaciones, percepciones y deseos detrás de cada acción de las participantes; y el análisis cualitativo de contenidos, que evidencia la interacción comunicativa en la plataforma.

En primer lugar, se realizaron entrevistas a profundidad a 14 mujeres jóvenes -de entre 17 y 27 años- integrantes del grupo de Facebook Ni Una Menos: Movilización Nacional Ya, que fueron divididas en dos grupos:
- Mujeres integrantes del grupo que no publicaron testimonios - lectoras (8 personas)

- Mujeres integrantes del grupo que publicaron testimonios - testimoniantes (6 personas)

Esta clasificación permitió dar cuenta de los diversos procesos comunicativos existentes, según cada papel y su relación con la plataforma. Además, se realizó una entrevista extra con una de las moderadoras del grupo, rol que ejercía controlando la admisión de miembros y aprobando las publicaciones.

Debido al carácter sensible de la investigación, las entrevistadas fueron contactadas a través del modelo de «bola de nieve», por el cual la persona entrevistada refería a otra integrante del grupo, esta a otra, y así sucesivamente. El único criterio de selección establecido fue que hubieran participado en el grupo de Facebook durante el periodo analizado. De forma posterior, se observó que todas las entrevistadas cumplían un perfil similar: estudiantes universitarias de una institución privada, de clase media y media alta de Lima y Callao.

En segundo lugar, se llevó a cabo el análisis de contenidos virtuales a través de los datos virtuales obtenidos en la comunidad de Facebook, con la finalidad de complementar las declaraciones de las entrevistadas y hacer un análisis descriptivo a partir de la exploración de las interacciones comunicacionales registradas 
en el portal. Con miras al estudio de este material, se solicitó permiso a todas las testimoniantes (6) para analizar la publicación de su testimonio y las interacciones que se generaron a partir de este.

Cabe precisar que el periodo de estudio de este grupo va desde su creación en Facebook, en julio de 2016, hasta dos meses pasada la movilización Ni Una Menos (NUM), es decir, septiembre de 2016. Sin embargo, el grupo sigue existiendo hasta el día de hoy, en 2020. Es necesario tener en cuenta que esta investigación no pretende ser representativa, pues el alcance del estudio es de una población limitada entre la totalidad de las integrantes del grupo -más de 40000 personas-.

\section{Facebook, plataforma determinante para la construcción de comunidad}

Facebook como plataforma, junto con la configuración de grupo cerrado, fueron determinantes para la consolidación de esta comunidad. Que fuera un grupo cerrado hacía que solo las personas previamente aprobadas pudieran ingresar y ver la lista de miembros y el contenido del grupo, lo cual generó una sensación de privacidad, seguridad y confianza emanada desde el apoyo expresado en los comentarios frente a las revelaciones sensibles: «Lo que hacía este espacio seguro eran los comentarios de apoyo» (Lectora 3). Esta sensación de seguridad fue res- guardada por las moderadoras, quienes establecieron reglas dentro del grupo para la interacción, como el creerle a las testimoniantes, no justificar agresiones, no juzgar a las víctimas, etcétera. Igualmente, se fue cuidando la integridad de las reveladoras: se eliminaba del grupo a las personas que faltaran a las normas y se borraban sus comentarios.

A pesar de esto, hubo varios problemas con la seguridad, ya que muchos testimonios fueron filtrados fuera del grupo a través de capturas de pantalla ${ }^{5}$ y compartidos en otros espacios; incluso, llegaron a manos de los agresores, lo que causó perjuicios a las víctimas.

Ello nos lleva a reflexionar sobre la seguridad efectiva de este espacio virtual, debido a que, como señalan Bazarova et al. (2015), los sitios de red social carecen de herramientas de gestión preventiva contra la divulgación, de modo que se arriesga la información personal de las usuarias.

Otra de las características mostradas fue la importancia de que las integrantes del grupo fueran usuarias frecuentes de Facebook y, por tanto, estuvieran familiarizadas con la interactividad, la inmediatez y la sensación de anonimato que genera estar detrás de una pantalla (García Galera et al., 2014); estaban acostumbradas a volcar su vida privada y sus pensamientos en estos portales, y hacerlos públicos.

\footnotetext{
${ }^{5}$ Una captura de pantalla es una fotografía a la pantalla que guarda la publicación observada. Permite que sea archivada como foto; después, puede ser enviada o transmitida en otras plataformas.
} 
Otra particularidad fue el patrón de repetición: las personas se animaban a compartir, repostear o sumarse a iniciativas y desafíos. De este modo, se observa cómo la publicación de testimonios sigue también esta lógica e impulsa a las mujeres a contar también sus experiencias. Lo comentó la Lectora 2: «Y con que una persona haya tenido el valor [...] todas las demás se animaron»; y se generó un efecto en cadena. Esto se explica, además, por el deseo de participar en una experiencia emocional compartida (Andalibi et al., 2018; Bazarova et al., 2015).

Ahora bien, un hallazgo importante fue la falta de representación de la comunidad LGTBIQ+: «Fue un espacio únicamente heterosexual» (Lectora 8). Fueron mínimos los casos de personas con otras orientaciones sexuales que compartieron sus testimonios, lo que limitó la exposición de casos de violencia, en una amplia mayoría, al modelo hombre agresor - mujer víctima.

En función de lo mencionado, es importante evidenciar la composición del grupo, ya que, a pesar de que la comunidad logró traspasar los límites locales, este estuvo conformado por personas relacionadas a través de círculos de contactos y conexiones, de estratos socioeconómicos similares que, además, tenían cierto interés o sensibilidad por el tema. Se comprueba, así, que los grupos en estas plata- formas, usualmente, son un reflejo de las relaciones sociales en la vida offline.

Dicho lo anterior, se debe prestar atención al privilegio que representa la participación en la esfera online, considerando la amplia brecha tecnológica y de uso que existe en el Perú. Lamentablemente, las personas más necesitadas de organización y agencia son las mismas que se encuentran relegadas del ámbito tecnológico, y es ahí donde se encuentra el potencial de este proceso: en la democratización de la participación colectiva.

Finalmente, se observa en esta comunidad virtual que hubo un periodo en el que la revelación de testimonios fue constante y en el que el contenido sensible fue el que forjó un ambiente de apoyo, entendimiento y sororidad 6 . Sin embargo, una vez pasada la marcha, y con el descenso del número de testimonios revelados, se gestó un espacio más racional, en donde primó la crítica y en donde se iniciaron conflictos respecto a temas como el aborto, que expusieron posturas, juicios y recriminaciones.

\section{La interacción comunicativa, desde la revelación sensible}

En cuanto a las dinámicas de interacción comunicativa desde el proceso de revelación, podemos decir que hubo múltiples y diversas formas de interactuar, pero que,

${ }^{6}$ «Relación de solidaridad entre mujeres, especialmente en la lucha por su empoderamiento» (Real Academia Española y Asociación de Academias de la Lengua Española, s. f., definición 2). 
en todos los casos, la plataforma virtual y $\mathrm{su}$ formato siguieron cumpliendo un papel determinante. Así, por ejemplo, cada participante replicó el modelo de interacción al que estaba acostumbrada; algunas fueron más activas y participativas que otras: «Yo solo miraba», señaló la Lectora 2, mientras que otras comentaban, compartían e invitaban a otras personas a unirse al grupo. No obstante, debido a la experiencia emocional compartida, las mujeres usualmente poco activas en estas plataformas se atrevieron a testimoniar.

Otra característica clave que marcó la forma de interacción fue la comunicación asincrónica (Andalibi y Forte, 2018), ya que, al no encontrarse atada al tiempo ni al espacio, se generó una mayor libertad en el modo de relacionamiento con la plataforma y el contenido sensible ahí publicado, lo que permitió que las usuarias pudieran interactuar a su ritmo, acercándose al grupo para escribir o leer, pero también alejándose para reflexionar. Se vieron, por ejemplo, modos de interacción en los que las entrevistadas mencionaban que «no podían dejar de leer» o «se pegaban» [a la pantalla], sin importar el lugar o momento en que se encontraran: «Ah, no, yo era una loca; yo andaba en el micro leyendo testimonios, o sea, a todos lados con mi celular. Yo leía, leía» (Testimoniante 4). De modo contrario, hubo quienes tomaron decisiones en relación con dónde y cuándo interactuar con el contenido. Por ejemplo, la Lectora 7 señaló: «Leía en mi casa, en mi intimidad. No me sentía cómoda de hacerlo en un lugar público
[...]. Siento que atentaba en contra de ese respeto que debería brindar». Este tipo de interacción, además, fue muy significativo para las mujeres testimoniantes, pues se observa la importancia del espacio en donde cada una repiensa y articula su vivencia, el espacio de enunciación: «Es super distinto escribirlo desde tu casa, sin mirar a las personas. $Y$, aunque vas a publicarlo frente a 10 ooo personas, no lo sientes como tan así» (Testimoniante 1). Ello implica atravesar el proceso de forma personal, en un espacio seguro, en la intimidad del hogar o de la propia habitación, lo que favorece la autorreflexión y el análisis: «Hay un montón de poder en escribir algo desde su propia casa» (Lectora 7). En definitiva, este recurso - que debe seguirse estudiando en futuras investigaciones para determinar su alcance y utilidad en el trabajo de temas complejos o sensibles - genera amplias posibilidades hacia un nuevo modo de interacción.

Respecto al proceso de decisión de revelar o no un testimonio en la plataforma, las mujeres debieron sopesar diversos factores -algunos de ellos relacionados con la audiencia, la red o la temporalidad-para decidir si participaban o no. Aquellas personas que se abstuvieron de revelar sus historias señalaron que se sentían limitadas por las características del grupo, la falta de anonimato, el alcance del mensaje, la presencia de alguna persona no grata en la comunidad, la desconfianza, o, simplemente, el hecho de no sentirse preparadas o deseosas de compartir información delicada en ese portal. No obstante, 
las entrevistadas que no testimoniaron también mencionaron el sentimiento de representación a través de otras historias reveladas, lo que evidencia una fuerte identificación colectiva: «Sentí que yo no iba a contar una historia distinta. Sentí que mis historias de acoso estaban siendo contadas por otras voces» (Lectora 3 ).

Ahora bien, con respecto a las mujeres que decidieron testimoniar, este espacio fue muy importante: «Es como decir "no me defienden acá; las autoridades no me prestan atención, pero ustedes sí me van a prestar. Me siento comprendida por ustedes"» (Testimoniante 3). En la modalidad del testimonio, es vital tener en cuenta que toda persona necesita encontrar una escucha para poder relatar sus sufrimientos (Pollak, 1989/2006). Las entrevistadas manifestaron que este fue un espacio alternativo de soporte, como señaló la Lectora 2: «Un espacio para ser escuchadas» y sentirse comprendidas y apoyadas.

De este modo, entre los motivadores internos para compartir su vivencia, se encuentra la visibilización de sus historias para que otras se pudieran identificar con su experiencia: «Para que se den cuenta [...] que no solamente a ellas les pasó lo que les pasó» (Testimoniante 6). Otra testimoniante destacó el acto de escribir y publicar como una forma de reivindicarse: «Me di cuenta de que es una manera de hacerte justicia a ti misma» (Testimoniante 1); y fue también una forma de liberación, como señaló la Testimoniante lo había dicho a nadie. Ponerlo en Facebook fue una manera de liberarme».

Asimismo, para algunas, el proceso de escribir significó el reconocer el episodio y poder catalogarlo como violencia. Por ejemplo, la Testimoniante 4 afirmó: «Siento que fue como una terapia, el escribirlo, el darte cuenta». Se pudo reconocer como una mujer víctima, pero también como sobreviviente de violencia, lo que es parte del proceso de empoderamiento. Así, los principales motivadores que se encontraron, con relación a la teoría expuesta, fueron los siguientes: sanar, obtener apoyo, reconocer y honrar una experiencia (Andalibi y Forte, 2018).

Adicionalmente, existieron otros factores propulsores de la revelación sensible de forma online. Por ejemplo, el formato de expresión - de una a muchas-, que permite a las personas evitar un gran número de conversaciones personales dolorosas y desafiantes con otros (Craig y Wright, 2012). Otro factor fue el miedo a ser juzgadas o cuestionadas: hacer la revelación sensible en ese espacio en línea permite obtener validación y soporte masivo, dado el alcance del mensaje. Como señala Loney-Howes (2018), el reclamar la propia experiencia y que sea validada por los pares es significativo.

En definitiva, el soporte obtenido fue esencial en la dinámica comunicacional, como señaló la Lectora 2: «Tú contabas y todo el mundo te respondía [...], o sea, 125 extraños diciéndote “¿estás bien?”». Las 
muestras de afecto fueron apreciadas, se manifestó la sororidad entre mujeres y se observaron frases en los comentarios como «hermana, yo te creo» o «somos hermanas en el dolor», que muestran la identificación común y el soporte. Se daban aliento la una a la otra, sabiéndose parte de la comunidad y de la problemática y uniéndose a través de un vínculo emocional, como señaló la Testimoniante 2: «La gente te apoya; te dicen "te quiero" sin conocerte». Andalibi y Forte (2018) señalan que este es el factor que garantiza el bienestar emocional de las reveladoras; publicaciones tan íntimas debían ser respondidas, comentadas, likeadas ${ }^{7}$. Las reacciones de apoyo en medios sociales son primordiales, al ser canales visibles donde las personas buscan atención y validación, como señaló la Testimoniante 4: «En una cosa tan importante como esta, era importante recibir una respuesta virtual, ¿no?». Esta testimoniante contó cómo incluso su enamorado le hizo un comentario en su publicación. A pesar de que lo hizo también de forma offline, era necesario para ella que su apoyo sea expuesto en la plataforma virtual.

Por otro lado, resulta interesante que la mayoría de las mujeres entrevistadas señalaron que no sabían cómo reaccionar frente a los testimonios leídos, dado el impacto de recibir información tan sensible. Así, las personas tendían a responder a través de reacciones livianas (Andalibi y Forte, 2018); es decir, colocaban un me gusta, me enoja o me entristece y eran partícipes, pero sin involucrarse al punto de tener que formular un comentario. Como explicó la Lectora 4: «Eran situaciones bien densas que te dejaban sin palabras y yo solamente atinaba a ponerle un like». Efectivamente, «las palabras quedaban cortas» (Testimoniante 2).

En cuanto a los comentarios, también se dio un proceso interesante de identificación, como lo describió la Testimoniante 3: «Le mandé un comentario; no lo había pensado [...]. Fue como comentarme a mí misma». Las mujeres, al involucrarse para dar un consejo en alguna publicación, sienten también que es una forma de aconsejarse y ayudarse a sí mismas, como lo sugiere el principio de la terapia de autoayuda (Bazarova et al., 2015).

Por último, un hallazgo importante fue el respeto a los límites de la virtualidad. Las testimoniantes expresaron que no hubiera sido oportuno dar paso a comentarios o preguntas en el plano offline: «No quería que muchos amigos vengan a decir "oye, ya, pero hablemos. ¿Cómo te sientes?”. Iba a ser incómodo» (Testimoniante 1). Señalaron que no se sentían preparadas para poder tener conversaciones cara a cara sobre un tema tan íntimo; lo harían cuando se sintieran listas y con determinadas personas. Sucedía lo mismo en el caso de las lectoras que debían servir de soporte para alguna amiga: preferían hacerlo de modo virtual, usando, por ejem- 
plo, el recurso del inbox ${ }^{8}$. Es a través de los mensajes privados o la bandeja de entrada que las personas comparten mensajes más íntimos.

Dicho lo anterior, a diferencia de la premisa del ciberactivismo, en que las acciones online deben pasar a una esfera offline para tener un impacto, en el caso de la revelación de contenido sensible, no se espera que los comentarios de apoyo y el cariño manifestados en la plataforma pasen a una esfera presencial.

\section{Impacto: concientización y empoderamiento}

El proceso de testimoniar fue en sí mismo relevante, ya que la persona que enuncia toma la palabra y constituye un saber del que antes no se tenía registro; debe repensarlo, volver a él, para poder constituirlo, darle sentido y hacerlo decible (Rodríguez, 2013). Hay poder en este proceso individual, que, al ser compartido, genera también que otras personas se vean reflejadas en estas historias.

Ello ocurre gracias a diversos mecanismos propios de esta experiencia virtual. Uno de ellos es el modo de lectura en primera persona, en el que el yo/tú (Yúdice, 1992) se superponen y generan identificación: «Yo había normalizado totalmente, $y$, viendo los testimonios, hago clic y digo: acá hay cosas que sí me han pasado» (Testimoniante 4). $\mathrm{O}$ el caso de la
Testimoniante 1, quien señaló: «Leyendo, escribiendo, llorando [...] ahí me acordé de lo que yo había vivido».

Este ha sido uno de los principales logros de la plataforma: que las mujeres tomen conciencia de la problemática a través de identificar que las vivencias de cada mujer no son casos aislados, como señaló la Testimoniante 4 respecto a los testimonios: «Abrieron esa posibilidad para decir: aguanta, ¿dónde estamos paradas? ¡Todas han pasado violencia!». Esto significa reconocerse parte de, abrir los ojos frente a una realidad que estaba silenciada y que es cotidiana.

Como resultado del reconocimiento de la problemática y la toma de conciencia, las participantes manifestaron modificaciones en sus actitudes y conductas personales. Por ejemplo, la Lectora 5 señaló: "Ahora me meto. Cuando me dicen algo en la calle, siempre respondo, reacciono. Siento ahí un gran cambio; me dio poder en mí misma». Por el lado de las testimoniantes, todas reafirmaron que el compartir su testimonio fue una decisión positiva, debido al soporte recibido, el poder reafirmar sus posturas, el no sentirse culpables, poder hacer decible su vivencia e, incluso, buscar apoyo, iniciando un proceso de sanación y empoderamiento. Por ejemplo, la Testimoniante 1 compartió que, después del proceso en el grupo de Facebook Ni Una Menos: Movilización Nacional Ya, no solo le comentó su vivencia a 
su familia por primera vez, sino que, además, comenzó a ir a terapia psicológica.

Vemos cómo se fortalece su autoimagen, considerando el reconocimiento de derechos, el aumento de su autoconfianza, su capacidad de respuesta frente a la violencia, entre otros.

Otra manifestación del empoderamiento individual es que las mujeres reconocieron el impacto que traían sus acciones en el contexto social más amplio, que se comprueba cuando las entrevistadas dijeron que fortalecieron su compromiso con la causa después de su experiencia en el grupo: «Enfatizó mi interés por querer pertenecer a estos movimientos» (Lectora 4); o, como señala la Testimoniante 4: «Para mí, sí ha sido como un antes y un después. Ahora quiero hacer un proyecto de mujeres».

En esta línea, el movimiento dejó un compromiso grande en muchas mujeres. Algunas, incluso, decidieron unirse a grupos militantes en contra de la violencia, como el caso de la Lectora 7: «Me motivó a luchar mucho más. Ahora milito en Colectiva9 ${ }^{9}$.

A nivel comunitario, otro logro del grupo fue su capacidad integradora, como comentó la Lectora 2: «Chocarte cara a cara con lo que estaba pasando generó una unión entre mujeres nunca antes vista». La Lectora 5, asimismo, señaló: «Descubrí el significado de la palabra sororidad».
Se observó cómo personas hasta ese momento extrañas enviaron mensajes a las víctimas para impulsarlas a denunciar, para ofrecerles apoyo psicológico o algún servicio en particular, como señaló la Testimoniante 3: «Psicólogas me daban consejos o abogadas me decían "oye, esta figura legal creo que todavía se puede ver”».

Así, se inició un amplio despliegue de iniciativas de solidaridad, como el apoyo en red, informativo y tangible (High y Buehler, 2019). Llamaron la atención los pedidos de auxilio en tiempo real, a los cuales las integrantes del grupo respondieron de forma inmediata - lo que evidenciaba su deseo de ayudar de forma presencialpreguntando por su ubicación y coordinando a través de mensajes internos. No obstante, debido al alcance de esta investigación, no se ahondó en la ayuda que se brindó en la esfera offline, que es un tema que requiere profundización para conocer su impacto.

A nivel social, si bien el inicio de la movilización es desencadenado por una chispa de indignación (Castells, 2012), el fenómeno de la revelación de testimonios en la comunidad virtual avivó la indignación de la población y, por tanto, el deseo de participar en el activismo por la causa.

A pesar de los conflictos que se dieron a nivel interno entre las organizadoras principales de la movilización (Caballero Rojas, 2018), se gestaron múltiples inicia- 
tivas a través de la organización comunitaria en barrios y distritos. Así, muchas de las entrevistadas, tanto lectoras como testimoniantes, participaron por primera vez en estos espacios de activismo presencial, en las asambleas abiertas -espacios autoconvocados-, en actividades artísticas de concientización, uniéndose a colectivos, entre otros: «Gente que nunca ha sido activa empezó a hacer cosas, a moverse, a hacer stickers, desde cosas chiquitas [...] dentro de sus posibilidades [...]. Rescato que la gente se movió. Fue un sacudón para la sociedad» (Testimoniante 4).

En el caso de las entrevistadas, participaron en diversas iniciativas, grupos de abogadas, grupos de discusión, grupos universitarios o, incluso, en acciones artísticas, como muralizaciones o pintas, y se involucraron en la toma de acción. En ese sentido, todas las entrevistadas afirmaron haber asistido a la marcha Ni Una Menos del 13 de agosto de 2016. Para muchas, esa fue su primera experiencia en una movilización.

En síntesis, a partir del análisis de esta comunidad virtual, se ha evidenciado la relevancia del proceso testimonial, clave para el reconocimiento de la problemática, tanto a nivel individual como colectivo. En él, las participantes identificaron sus propias historias en las de otras mujeres, y percibieron la cercanía de la problemática y su carácter colectivo. Este proceso, si bien causó tristeza, se condensó en forma de ira, y generó indignación y la convicción de la necesidad de hacer algo al respecto, de modo que las frustraciones individuales se transformaron en una fuerza colectiva.

\section{A manera de conclusión: algunas luces y cuestionamientos}

Hemos observado, en los últimos años, el poder de la agrupación y organización colectiva que surge de la utilización del nuevo espectro tecnológico - en especial, de los sitios de red social-y que se evidencia en movimientos activistas con un fuerte impacto a nivel local y global por intermedio de sus estrategias hacia el salto offline.

En Ni Una Menos: Movilización Nacional Ya, el grupo de Facebook estudiado, la organización de miles de mujeres fue potenciada por un proceso de revelaciones sensibles que resultó en la marcha más multitudinaria de mujeres en el Perú, un precedente importante que colocó el tema en la agenda pública. Así, este no fue un simple caso de activismo, de coordinación online y su paso al espacio offline, sino que fue el resultado de un fenómeno de interacción comunicativa desde la revelación sensible, que caló a nivel individual y colectivo en una comunidad de miles de mujeres.

En esta investigación, se profundizó en las dinámicas comunicacionales internas del grupo, que evidenciaron el proceso de apropiación de la plataforma web por parte de las usuarias, ya que un espacio que originalmente fue destinado para el activismo se convirtió en un lugar de denun- 
cia frente a la necesidad de las mujeres de relatar sus historias silenciadas y almacenadas en las memorias individuales.

Fue así como este portal se convirtió en un lugar de encuentro, un espacio seguro donde las mujeres se apoyaron unas a otras. Sirvió de soporte y fue la escucha que necesitaban como medio de reivindicación colectiva.

En ese sentido, es relevante resaltar la importancia del periodo de revelación de testimonios que ocurrió en la plataforma; en esta, el contenido sensible forjó un ambiente de entendimiento. Es preciso observar en el grupo el poder de la enunciación, desde la emoción y la apertura, para la concientización a través de la empatía, antes de volverse un espacio racional y argumentativo. Este punto debe ser recuperado por futuras investigaciones como estrategia metodológica para el logro de procesos de sensibilización en diversas esferas.

Se sugiere, además, profundizar en cómo cambian las dinámicas de interacción entre los niveles online y offline, en vista de que se presentan otro tipo de códigos, expectativas y límites, como lo sugieren, por ejemplo, las testimoniantes entrevistadas al revelar sus vivencias en este portal.

Por otro lado, se pone en cuestión, también, la respuesta que se da frente a la revelación de testimonios online, proceso que sigue produciéndose y que cada vez genera mayor debate en cuanto a la cre- dibilidad o al riesgo de difamación de los presuntos agresores. Sin embargo, no se hace mucho hincapié en esta herramienta como proceso de reivindicación, de denuncia, o de acceso a justicia y reparación. Deben repensarse respuestas formales frente a la cada vez mayor expresión de contenido sensible en estas plataformas. Institucionalmente, pueden abrirse canales que aprovechen las características de estos medios sociales y tomen en cuenta las necesidades de la población.

Por último, queda evidenciado cómo se genera un proceso de empoderamiento desde la revelación sensible de relatos, que reclaman su existencia y quiebran una realidad dominante, lo que permite la identificación de la problemática. En lo más profundo y de manera individual, las mujeres no solo se sienten validadas y acompañadas, sino que comienzan a generar acciones en sus relaciones cercanas, en sus vínculos, en el espacio público, y en la defensa de sí mismas y de otras mujeres.

Este proceso surge desde el empoderamiento comunitario, desde la articulación y la generación de vínculos de solidaridad, identificación y reconocimiento, pero también desde los sentimientos de indignación que motivaron la organización colectiva. Se observó en las entrevistadas un creciente deseo de cooperación, de compromiso con la causa, que las motivó a participar, incluso por primera vez, en espacios de organización colectiva y activismo. 
De este modo, el proceso aquí analizado calza con la teoría, pues la interacción comunicativa fue determinante para la toma de conciencia y organización desde el proceso de empoderamiento individual y colectivo. Se impulsa a otros investigadores e investigadoras a profundizar en estas dinámicas online, que, si bien funcionan de manera similar en un entorno offline, cuentan con otras características y recursos propios de la virtualidad que facilitan el proceso y que pueden ser aprovechados.

Se sugiere, entonces, una ampliación de la comprensión del proceso de empoderamiento desde la teoría de la comunicación para el cambio social y el paradigma participativo en la literatura académica, considerando su potencial en el entorno social digital, debido a que, en su mayoría, los estudios se han centrado en entornos físicos y comunitarios.

Finalmente, con el presente trabajo de investigación, se evidencia la importancia de los procesos comunicativos en el espectro tecnológico y su relevancia para grupos que, como las mujeres, continúan articulándose y resistiendo, e impulsando el cambio social.

\section{REFERENCIAS}

América Noticias (2016, 15 de julio). Ayacucho: agresor que golpeó a mujer en hostal fue excarcelado esta tarde. https://www.americatv.com.pe/ noticias/actualidad/agresor-ayacucho-fue-puesto-libertad-golpiza-joven-hotel-n239131

Amichai-Hamburger, Y., McKenna, K. Y. A. y Tal, S.-A. (2008). E-empowerment: Empowerment by the Internet. Computers in Human Behavior, 24(5), 1776-1789. https://doi.org/10.1016/j. chb.2008.02.002

Andalibi, N. y Forte, A. (2018). Responding to sensitive disclosures on social media: A decision-making framework. ACM Transactions on Computer-Human Interaction, 25(6), Artículo 31. https://doi.org/10.1145/3241044

Andalibi, N., Haimson, O. L., De Choudhury, M. y Forte, A. (2018). Social support, reciprocity, and anonymity in responses to sexual abuse disclosures on social media. ACM Transactions on Computer-Human Interaction, 25(5), Artículo 28. https://doi. org/10.1145/3234942

Bazarova, N. N., Choi, Y. H., Schwanda Sosik, V., Cosley, D. y Whitlock, J. (2015). Social sharing of emotions on Facebook: Channel differences, satisfaction, and replies. En D. Cosley, A. Forte, L. Ciolfi y D. McDonald (Eds.), CSCW '15: Proceedings of the 18th ACM Conference on Computer Supported Cooperative Work \& Social Computing (pp. 154-164). Association for Computing Machinery. https:// doi.org/10.1145/2675133.2675297 
Blanchard, A. y Lynne, M. (2004). The experienced «sense» of a virtual community: Characteristics and processes. ACM SIGMIS Database: The DATABASE for Advances in Information Systems, 35(1), 64-79. https://doi. org/10.1145/968464.968470

Butler, B. S., Bateman, P. J., Gray, P. H. y Diamant, E. I. (2014). An attraction-selection-attrition theory of online community size and resilience. MIS Quarterly, 38(3), 699-728. https://doi. org/10.25300/MISQ/2014/38.3.04

Caballero Rojas, G. A. (2018). Redes sociales y feminismos en la acción colectiva: el caso de «Ni Una Menos» en el Perú [Tesis de maestría, Pontificia Universidad Católica del Perú]. Repositorio Digital de Tesis y Trabajos de Investigación PUCP. http://hdl.handle. net/20.500.12404/11944

Cadiz, M. C. (2005). Communication for empowerment: The practice of participatory communication in development. En O. Hemer y T. Tufte (Eds.), Media and glocal change: Rethinking communication for development (pp. 145-158). CLACSO.

Cano Isaza, T. A. y Arroyave Álvarez, O. A. (2014). Procesos de empoderamiento de mujeres: subjetivación y transformaciones en las relaciones de poder. Revista Virtual Universidad Católica del Norte, (42), 94-110. http://revistavirtual.ucn.edu.co/index.php/RevistaUCN/article/view/497/1033

Carr, C. T. y Hayes, R. A. (2015). Social media: Defining, developing, and divining. Atlantic Journal of Communication, 23(1), 46-65. https://doi.org/10.108o/ 15456870.2015.972282

Castells, M. (2012). Redes de indignación y esperanza. Los movimientos sociales en la era de internet (Trad. M. Hernández). Alianza Editorial.
Craig, E. y Wright, K. B. (2012). Computer-Mediated relational development and maintenance on Facebook ${ }^{\circledR}$. Communication Research Reports, 29(2), 119-129. https://doi.org/10.1080/0882 4096.2012.667777

García Galera, M. C., Del Hoyo Hurtado, M. y Fernández Muñoz, C. (2014). Jóvenes comprometidos en la Red: el papel de las redes sociales en la participación social activa. Comunicar, 22(43), 35-43. https://doi.org/10.3916/C432014-03

Garzón Martínez, M. A. (2015). La subjetividad rememorante. Revista Colombiana de Sociología, 38(2), 115-137. https:// doi.org/10.15446/rcs.v38n2.54902

Gerbaudo, P. (2012). Tweets and the streets: Social media and contemporary activism. Pluto Press. http://library.oapen. org/handle/20.500.12657/30772

Gil Piedra, R. (2017). Del Nunca Más al Ni Una Menos: Memoria, violencia y comunidad en Perú. Revista Argumentos, (2), 5-9. https://revistaargumentos.iep.org.pe/wp-content/ uploads/2017/10/GIL_11_2_2017.pdf

Gumucio-Dagron, A. (2002, 18-20 de septiembre). Comunicación para el cambio social: clave del desarrollo participativo [Ponencia magistral de apertura]. XX Encuentro Académico de AFACOM: Comunicación, Educación, Ciudadanía, Medellín, Colombia. http://revistas.javeriana.edu.co/ index.php/signoypensamiento/article/viewFile/2454/1728 (Trabajo original publicado en 2001)

Hernández Sampieri, R., Fernández Collado, C. y Baptista Lucio, M. d. P. (2010). Metodología de la investigación (5. ${ }^{\text {a }}$ ed.). McGraw Hill. (Trabajo original publicado en 1991) 
High, A. C. y Buehler, E. M. (2019). Receiving supportive communication from Facebook friends: A model of social ties and supportive communication in social network sites. Journal of Social and Personal Relationships, 36(3), 719-740. https://doi. org/10.1177/0265407517742978

Hsiu-Fen, L. (2007). The role of online and offline features in sustaining virtual communities: An empirical study. InternetResearch, 17(2), 119-138.https:// doi.org/10.1108/10662240710736997

Instituto Nacional de Estadística e Informática. (2016, octubre). Encuesta Nacional sobre Relaciones Sociales ENARES 2013 y 2015 (principales resultados). https://www.inei.gob.pe/ media/MenuRecursivo/publicaciones_digitales/Est/Lib139o/libro.pdf

Instituto Nacional de Estadística e Informática. (2020, junio). Estadísticas de las Tecnologías de Información y Comunicación en los Hogares. Trimestre: Enero-Febrero-Marzo 2020, (02). https:// www.inei.gob.pe/media/MenuRecursivo/boletines/boletin_tics.pdf

Loney-Howes, R. (2018). Shifting the rape script: «Coming out» online as a rape victim. Frontiers: A Journal of Women Studies, 39(2), 26-57. https://doi.org/10.5250/fronjwomestud.39.2.0026

Mendivil Calderón, C., Racedo Durán, Y., Meléndez Solano, K. y Rosero Molina, J. (2015). El papel de la Comunicación para el cambio social: empoderamiento y participación en contextos de violencia. Revista Encuentros, 13(1), 11-23. http://ojs.uac.edu. co/index.php/encuentros/article/ view/345/309
National LGBT Health Education Center. (2018). Glosario de términos LGBT para equipos de atención a la salud (Trads. Departamento de Servicios de Intérpretes de Vanderbilt University Medical Center: N. Griffin, L. Albritton y D. Mejía). https:// www.lgbthealtheducation.org/ wp-content/uploads/2018/03/National-LGBT-Health-Education-Center-Glossary.SPANISH-2018.pdf

Ochman Ikanowicz, M. B. (2016). Políticas sociales y empoderamiento de las mujeres. Una promesa incumplida. Estudios Políticos, (48), 32-51. https:// doi.org/10.17533/udea.espo.n48ao3

Petrič, G. y Petrovčič, A. (2014). Individual and collective empowerment in online communities: The mediating role of communicative interaction in web forums. The Information Society, 30(3), 184-199. https://doi.org/10 $.1080 / 01972243.2014 .896683$

Pollak, M. (2006). Memoria, olvido, silencio. La producción social de identidades frente a situaciones límites (Trads. C. Gebauer, R. Oliveira y M. Tello). Ediciones Al Margen. (Trabajo original publicado en 1989)

Real Academia Española y Asociación de Academias de la Lengua Española. (s. f.). Sororidad. En Diccionario de la lengua española. Recuperado el 20 de julio de 2019 de https://dle.rae. es/?id=YQRfknk

Rodríguez, R. P. (2013). El poder del testimonio, experiencias de mujeres. Revista Estudios Feministas, 21(3), 11491169. https://doi.org/10.1590/So104026X2013000300021 
Soto Canales, V. (2019). El poder de la interacción comunicativa en comunidades virtuales: el caso del grupo de Facebook «Ni una menos, movilización nacional ya» [Tesis de licenciatura, Pontificia Universidad Católica del Perú]. Repositorio Digital de Tesis y Trabajos de Investigación PUCP. http://hdl.handle. net/20.500.12404/15059

Troncoso Pérez, L. E. y Piper Shafir, I. (2015). Género y memoria: articulaciones críticas y feministas. Athenea Digital, 15(1), 65-90. https://doi.org/10.5565/ rev/athenea.1231

Tufte, T. (2015). Comunicación para el cambio social. La participación y el empoderamiento como base para el desarrollo mundial. Icaria.

Yates, S. y Lockley, E. (2018). Social media and social class. American Behavioral Scientist, 62(9), 1291-1316. https:// doi.org/10.1177/0002764218773821

Yúdice, G. (1992). Testimonio y concientización. Revista de Crítica Literaria Latinoamericana, (36), 211-232. https:// doi.org/10.2307/4530631 